\title{
Study on Synthesis Method of Character Animation under the Perspective of Physics
}

\author{
Xuetao $\mathrm{Ma}^{1}$ \\ ${ }^{1}$ Luohe Medical College, Luohe, Henan, 462000 \\ a email
}

\begin{abstract}
Keywords: Character Animation; Physical Simulation; Motion Synthesis; Virtual Reality; Motion
\end{abstract} Control

\begin{abstract}
Animation and character animation has been one of important research field of virtual reality contents of the computer in recent years, with the vigorous development of 3D games and animation and film special effects industry, the role of the physical animation authenticity increasingly urgent demands, based on the physical character animation synthesis researchers have been more and more attention, spawned many new methods and new technologies. the core of the problem is the study of human motion synthesis method, it is intended to drive a virtual character motion, they produce the physical law of motion animation. focus on Advances in synthetic methods are described first in character animation for the whole domestic and foreign research and analysis and summary on the basis of calculations based on different joint torque will be divided into 7 categories: time and space constraint method, constrained dynamic optimization method, after the low-dimensional model, finite state machines, data-driven, kinetic filtration, probabilistic modeling method, described in detail the principles and characteristics of each class methods, focusing on new working methods in each class recent Secondly, the above comparative analysis of advantages and disadvantages of the method carried out. Finally, the actual application requirements, deficiencies exist in the work for the present, can continue to make some in-depth research.
\end{abstract}

\section{Introduction}

In recent years, with the continuous development of computer animation techniques, 3D game animation, film special effects showing a booming trend, the 21st century is one of the brightest character animation industry as a synthesis of these industries in the main contents, causing research staff attention which, based on the physical character animation because it can comply with the natural movement of the synthetic fragment action, computer animation is one of the most active research areas in recent years in the field.

\section{Physical Synthesis Method Based On Character Animation}

Physics-based character animation synthesis, which calculate a joint torque according to mission objectives, driving physics-based character motion. Character animation synthesis method is a virtual character motion in a virtual environment, the "brain" is the character animation system core content based on physical character animation synthesis methods, resulting in the trajectory planning and classical control methods in the 1980s are two kinds of classical synthetic methods. in recent years, the advent of computer animation field many new technologies and new methods

Based on the calculation method of character animation synthesis method different from the joint torques and development, especially the development of motion capture technology and advanced optimization methods, the maturity, the traditional method and the continuous integration of new technologies evolve many new character animation synthesis method, roughly the space is divided into task optimal control, joint space control methods and hybrid methods of physical and non-physical simulation model of three categories based on the physical character animation methods, and further divided into seven categories. this section first introduced each principle class methods, and processing methods to realize the difficulty, and then compared the performance of a 
list of all kinds of methods, and summarizes the advantages and limitations of various kinds of methods.

That space-time trajectory planning method optimization method is one of the seminal physical character animation synthesis method based on. Trajectory optimization method combines biomechanics rules and optimization techniques, the role of the action required to meet a variety of physical conditions are described in a variety of constraints function properly according to biomechanical principles character motion defined objective function, character animation synthesis problem into a respective concrete offline temporal optimization problem. the method in addition to guarantee the physical realism movement, but also can demand according to different actions modify the objective function and constraint functions, to a certain extent to achieve the control of the final animation of such methods in the actual application process mainly in the following three questions: 1) degree of freedom of role models and more computationally intensive optimization, offline Processed optimization; 2) Although you can adjust the objective function and constraint functions, to control the character animation purposes, but still can not control the real-time character animation; 3) optimization model of multi-variable, nonlinear strong, select the optimal solution for the initial value very sensitive, additional optimization model can not effectively deal with some of the high-level control parameters, such as walking speed.

Dynamics constrained optimization control method from optimization control theory, optimization model to calculate real-time control tasks to meet the high level of human joint torque via multi-objective driven body movement. The biggest difference between this method and the method optimization trajectory is calculated trajectory Optimization in the entire movement sequence, but with constrained multi-objective optimization method is to optimize the calculation of the time or motion clip short time as optimization period of high jump action, trajectory optimization method smooth adjustment of all motion sequence centroid height, and multi-objective optimization rule with constraints the frame is to adjust the torque and knee lift heel to centroid expected to meet the current frame height constraint of the technical difficulties of the method implemented include: 1) more freedom because the body joints, the joint range of motion quite different and difficult to control, the biggest difficulty is that dynamic constraints to optimize real-time control method to solve the optimization model; 2) local optimization model, the objective function typically involves biomechanics items, balance controls, style controls, motion tracking items, and even parameter controls, multi-objective optimization is not easy to solve.

Low-dimensional physical model method commonly used first-order inverted pendulum, spring pendulum and other low-dimensional physical model simulation role global motion information, after obtaining the overall motion information, combined with human motion characteristics and user parameter control, synthesis of body movement. in the field of synthetic character animation, character models generally use more rigid tree structure describes the kinetic equation is a nonlinear control system of a multi-variable and difficult to control. researchers try to use to reduce the dimension of the high-dimensional nonlinear systems, and motion editing low-dimensional mathematical model is slightly different, dimension reduction mentioned here dimensionality reduction is not just movement variables, but the use of low-dimensional physical model for the overall message of human motion, and then combined with other control parameters and biomechanical principles to generate systemic movement of low-dimensional physical model method 2:00 important consideration in the implementation: 1) what kind of low-dimensional model to more accurately describe the role of the overall motion information; 2) based on low-dimensional physics model sports how can synthesize natural realistic human motion.

Finite state machine controller methods from classical control theory, it will be seen as the motion conversion process between the finite-state machine by setting a PD (proportional-derivative) controller at each joint, the control character movement from one state to the next state to synthesize a control character periodic motion. and the top 3 ways to optimize space in the task to calculate different joint torque, the controller method to calculate the joint torque in joint space using analytical equations, the controller input for the desired joint posture, joint control of the output torque. torque is calculated so that the controller of this analytical method 
to generate human motion efficiency is extremely efficient, capable of $1000 \mathrm{fp}$, speed real-time control synthesis of human movement. in addition, PD controller calculates the joint torques so that the the method is more robust, able to respond to outside interference in real time finite state machine controller method in the application of the major challenges which face the following: 1) the specific role and movement difficult to visually determine the appropriate force and torque control is difficult to design; 2) is less versatile, when the motion type or environment changes, you need to divide each of the finite state machine manual movement and re-adjust the parameters of the controller; classic 3) controller method from the robot control theory, the synthesis of animation operation more machinery.

Data-driven method, which is calculated based on the physical equations of motion joint torque driving role model to track the motion capture data to external interference or environmental changes to make real-time feedback. In recent years, with the motion capture technology has become more sophisticated and increasingly large database of motion capture, motion capturing data obtained in the character animation technology and methods in the more widely used, is playing an increasingly important role due to the motion capture data contains a wealth of details, natural and realistic, but based on physical methods generally natural authenticity poor, how to use motion capture data to improve the fidelity of synthesis of natural movement, is a research hotspot in recent years and are based on different kinematics editor synthesis algorithm, data-driven method here is not simply a motion stitching and redirection, but and motion capture method based on kinetic data, real-time calculation of joint torque, track has some motion capture data which has the following difficulties in implementation: 1) motion capture data is not consistent with a role model, both of which may contain errors; 2. ) in the motion capture data does not include the joint torque and the contact with the ground forces, the role of sports in the data-driven stages need to be recalculated.

\section{Summary and Future Research}

Since the dynamics of character animation system is a multi-variable, strong coupling, underactuated unstable system, combined with people for the role of sports in particular are very sensitive to human movement familiar with physics-based character animation has been in the field of computer animation difficulties through recent decades, especially the past 10 years of development and accumulation, the researchers around the above difficulties and key performance character animation done a lot of research work and achieved some research results. However, industrial applications, based on the physical character animation method is still relatively rare, physics-based character animation from a real industrialization still has a lot of distance.

In this section we first combine character animation difficulties, respectively from the controllability, balance control, motion and other animation style synthesis difficulties of summaries of physical synthesis method based on character animation. On this basis, it is based on physical the character animation in industrial applications encountered challenging problem, we can continue to make some in-depth study of the problem, and pointed out possible solutions.

Anime synthesis method based physical synthesis is the core of the animation, we need to take into account the three parties and factors: level parameter control, balance control, movement style we introduced research progress in these three areas in recent years.

Multi-body system variables and more physically based animation synthesis and each joint variable coupling exists, not only make the system very difficult to control, but also greatly reduce the controllability of the system, even when serious so that the system can not run. In addition, the role of the motion system is an under-drive system, the joint torques into contact with an unknown moment to joint control character movement, and outside the contact force is usually unpredictable, so the role of control is more difficult.

Ground contact model. To achieve the purpose of controlling the movement of the role, the first ground contact force and a mathematical model, the use of land and joint contact forces combined torque control character movement balance to the existing literature and the main contact force three methods described: 
Punishment law punish law allows light to penetrate to the role and then apply a spring-damper model is based on mutually exclusive, punishment is proportional to the penetration depth of force. This method is simple and convenient, but less robust, different simulation step long it will lead to a significant difference and contact force. in addition, on this model, strictly speaking not physically real.

Coulomb friction cone model. The model assumes that the contact force and ground by a group unit friction element restriction within one inverted cone. Unlike joint contact force constraint, is a unilateral restraint, and contact force can be determined by optimization methods.

Virtual force model (virtual force). In this model, Jacobian matrix and the contact force will be converted to the centroid coordinates, End

A high-level tasks while controlling the movement balance.

Physics-based character animation, calculated in accordance with its joint torque can be divided into synthetic Anime Anime joint space mission space synthesis and its motion control, the same can be done in both the joint space and to be in the task space get on.

Parameter controls the joint space. Joint space controller class correlation method, through feedback control theory, based on the current state and the target joint stance explicitly calculate a joint torque feedback outside interference. Because this control calculation method uses feedback joint torques of external disturbance and parameter perturbation has strong robustness, however, due to the strong coupling between the controller parameters, parameter level task space is not easy to precisely control, such as the stride, orientation, location and other extremities. Although C 'oro et al proposed different techniques for performing high-level control parameters, but its implementation process is complex, difficult to model for the animators to understand, limiting its application.

Parameters control tasks in space. Optimal Control class methods, the use of multi-objective optimization model calculates joint torque, the objective function can be added to high-level controls, which can rise well done interactive control parameters.

Compared to motion editing method, based on the physical character animation more difficult to control, which is a reason for the instability of multi-body systems in physics-based animation synthesis, it is very important that the need to keep the balance not to fall role pour role quiet than standing balance of animation generation, most of the literature is by controlling the centroid of the ground and of the projection so as to maintain control of balance and inner support. Since the momentum and center of mass acceleration has analytical relations, in addition to the literature by controlling momentum and angular momentum and thus control the balance.

In the motion synthesis, synthesis of early animation keyframe manually or set Chuan finite state machine control balance. In recent years, researchers have focused on the study of auto-adjust the balance controller, Yin et al., According to the current swinging leg position and the target position by feedback error learning controller of center of mass between the support leg transition .Lee and others have used similar ideas, feedback disturbance while tracking motion capture data. further, Abe, who will be human motion centroid and the press center relative position as the phase space tracking motion capture data with reference to the object, further increasing the robustness of the algorithm to maintain a balance.

Since most methods are by controlling the centroid position relative to the center of pressure, indirect control balance; and low-dimensional physical models directly control the centroid position, describe the overall motion information, it is based on the method of low-dimensional physical model is the best way movement balance control .Tsai et al. first-order inverted pendulum describe human walking changes in motion the center of mass, combined with motion capture data generated to meet customer control requirements walking motion while Kwon et al., using a first-order inverted pendulum model describes the body running the centroid changes above 2 are used in both the first-order inverted pendulum model to describe the motion of the center of mass of human motion, but since the model assumes that the inversed pendulum rod length remains constant during the entire movement, can not accurately describe the human lower limb movement. in order to improve the above problems, Mordatch et al. used a spring pendulum model describes human 
walking in the center of mass motion, balance control human movement.

Natural and realistic character animation nature, meaning there are two layers: 1) motion biomechanically as well as specific types of sports law, such as human motion acting minimum speed changes gently, walking in the same position of the head relative to the center of mass, approximately knee do not force the like; 2) the role of animation and real biological motion similar.

Early in the movie based on the physical role, since the motion capture data technology method of immature conditions, so most methods by optimizing the synthesized animation meet biomechanical laws to ensure their natural verisimilitude .Wample: biomechanics, who according to the law, the use of the track planning to generate a virtual prehistoric animals and animal movements, who acts .Wang optimize bio-mechanics finite state machine controller parameters, the controller generates an operation more in line with the movement of the human body.

In recent years, with the motion capture technology has become more sophisticated as well as motion capture large-scale database applications, more and more researchers by controlling the character animation and motion capture data close to indirect guarantee natural realism movement. Data-driven the method of ensuring authenticity while tracking physical motion capture data, increasing the animated nature verisimilitude.

\section{Conclusion}

As the field of computer animation character animation important research, has been involved in research content is very rich, is a cross-cutting areas of computer graphics, biomechanics, robotics, artificial intelligence, machine learning and other disciplines, widely used in sports simulation, Entertainment, 3D games, military simulation and other fields, the ultimate goal is to generate dynamic and full of character animation real close when this paper describes the recent literature about the main character animation methods, the main work in recent years were introduced by category, outlined their principles and advantages and disadvantages of methods; at the same time, with the actual application requirements, deficiencies exist in the work for the present, the future make some challenging problems, to solve these problems will lead to the continuous improvement of the quality of the generated character animation and animation efficiency, thus further promoting the character animation in-depth promotion and application in various fields.

\section{Acknowledgements}

This article is based in Henan Province Science and Technology Department forefront of technology research projects in 2014. Item number: 142300410340.

\section{References}

[1] Pan Zhigeng. (inaudible) and so on. Summary of machine learning techniques of human motion synthesis [J]. Computer Aided Design and Computer Graphics, 2010, 22 (9) 1619-1627)

[2] Xia Shihong, Wei Yi, Wang Zhaoqi. A survey o1 physicsbased human motion simulation [J]. Journal 01 ComputerResearch and Development, 2010, 47 (8) 1354-1361 (Chinese)

[3] Xiashi Hong, Wei Yi, Wang Zhaoxing reviewed its human motion simulation [J] Computer Research and Development, 2010, 47 (8): 1354-1361)

[4] Li Shilei, Liang Jiahong, Wu Bing, et al Survey o1 virtualcharacter motion generation and control [J] Journal ofSystem g Simulation, 2011,23 (9): 1758-1771 (in Chinese)

[5] Lishi Lei, Liang and red, Wu Bing, etc. generate virtual human motion and control technology Survey [J]. Journal of System Simulation, 2011

[6] Pan Zhigeng, Lii Pei, Xu Mingliang, et al. A survey on to wdimensional space expression for human motion data and character animation generation [J]. Journal 01 Computer-Aided Design Computer Uraphics, 2013,25 (12) 1775-1785 (in Chinese) 
[7] Pan Zhigeng, Lvpei, Xu Mingliang, and other low-dimensional human motion data-driven role [J] Computer Aided Design and Computer Graphics, 2013,26CL2\}:. 1775-1785)

[8] Wampler, K, Popovic Z. Optimal gait and form for animallocomotion [J]. ACM Trans on Uraphics 2009,28 (3) Article No 60. 